MATHEMATICS OF COMPUTATION

Volume 75, Number 256, October 2006, Pages 1905-1912

S 0025-5718(06)01868-0

Article electronically published on June 28, 2006

\title{
ON EFFICIENT COMPUTATION AND ASYMPTOTIC SHARPNESS OF KALANTARI'S BOUNDS FOR ZEROS OF POLYNOMIALS
}

\author{
YI JIN
}

\begin{abstract}
We study an infinite family of lower and upper bounds on the modulus of zeros of complex polynomials derived by Kalantari. We first give a simple characterization of these bounds which leads to an efficient algorithm for their computation. For a polynomial of degree $n$ our algorithm computes the first $m$ bounds in Kalantari's family in $O(m n)$ operations. We further prove that for every complex polynomial these lower and upper bounds converge to the tightest annulus containing the roots, and thus settle a problem raised in Kalantari's paper.
\end{abstract}

\section{INTRODUCTION}

In 3, Kalantari derives an infinite family of lower and upper bounds on the modulus of zeros of analytic functions. When applied to complex polynomials, these bounds can be computed in polynomial time.

Let

$$
f(z)=a_{n} z^{n}+a_{n-1} z^{n-1}+\cdots+a_{1} z+a_{0}, a_{0}, \ldots, a_{n} \in \mathbb{C}, a_{n} a_{0} \neq 0,
$$

and

$$
F(z)=f(z) z
$$

Then from an expansion formula of a fundamental family of root-finding iteration functions applied to $F(z)$ at $z=0$ (see (2.4) in [3]), we get

$$
1+\sum_{i=1+k}^{n+k}(-1)^{k+2} \frac{\hat{D}_{k+1, i+1}(0)}{D_{k+1}(0)} \theta^{i}=0
$$

Received by the editor January 14, 2005 and, in revised form, August 3, 2005.

2000 Mathematics Subject Classification. Primary 12D10, 65H05, 68Q25; Secondary 05A15, $11 \mathrm{~B} 37$.

Key words and phrases. Bounds on polynomial roots, asymptotic sharpness, generating functions, recurrence relations.

(C)2006 American Mathematical Society Reverts to public domain 28 years from publication 
for $k=0,1,2, \ldots$, where $\theta$ is a zero of $f(z)$, and

$$
\begin{array}{r}
D_{m}(z)=\operatorname{det}\left(\begin{array}{ccccc}
F^{\prime}(z) & \frac{F^{\prime \prime}(z)}{2 !} & \cdots & \frac{F^{(m-1)}(z)}{(m-1) !} & \frac{F^{(m)}(z)}{(m) !} \\
F(z) & F^{\prime}(z) & \cdots & \ddots & \frac{F^{(m-1)}(z)}{(m-1) !} \\
0 & F(z) & \ddots & \ddots & \vdots \\
\vdots & \vdots & \ddots & \ddots & \frac{F^{\prime \prime}(z)}{2 !} \\
0 & 0 & \cdots & F(z) & F^{\prime}(z)
\end{array}\right), \\
\hat{D}_{m, j}(z)=\operatorname{det}\left(\begin{array}{ccccc}
\frac{F^{\prime \prime}(z)}{2 !} & \frac{F^{\prime \prime \prime}(z)}{3 !} & \cdots & \frac{F^{(m)}(z)}{(m) !} & \frac{F^{(j)}(z)}{j !} \\
F^{\prime}(z) & \frac{F^{\prime \prime}(z)}{2 !} & \ddots & \frac{F^{(m-1)}(z)}{(m-1) !} & \frac{F^{(j-1)}(z)}{(j-1) !} \\
F(z) & F^{\prime}(z) & \ddots & \vdots & \vdots \\
\vdots & \vdots & \ddots & \frac{F^{\prime \prime}(z)}{2 !} & \frac{F^{(j-m+2)}(z)}{(j-m+2) !} \\
0 & 0 & \cdots & F^{\prime}(z) & \frac{F^{(j-m+1)}(z)}{(j-m+1) !}
\end{array}\right),
\end{array}
$$

and "det" denotes determinant.

Let

$$
\hat{c}_{k, i}=(-1)^{k+2} \frac{\hat{D}_{k+1, i+1}(0)}{D_{k+1}(0)}
$$

then (1.1) becomes

$$
1+\sum_{i=1+k}^{n+k} \hat{c}_{k, i} \theta^{i}=0
$$

Now define

$$
\gamma_{k}=\max \left\{\left|\hat{c}_{k, i}\right|^{1 / i}: i=1+k, \ldots, n+k\right\} \quad \text { and } \quad u_{k}=\gamma_{k}|\theta| .
$$

Then $u_{k}^{i} \geq\left|\hat{c}_{k, i} \theta^{i}\right|$ and

$$
\sum_{i=1+k}^{n+k} u_{k}^{i} \geq \sum_{i=1+k}^{n+k}\left|\hat{c}_{k, i} \theta^{i}\right| \geq 1 .
$$

Proposition 1.1. Inequality (1.3) implies $u_{k}>r_{k}$, where $r_{k}<1$ is the unique positive zero of the polynomial $t^{k+1}+t-1$.

Proof. The following proof was given by Kalantari [3].

First, the polynomial $q(t)=t^{k+1}+t-1$ has a unique positive zero $r_{k}<1$. This follows from the inequalities $q(0)<0, q(1)>0$, and $q^{\prime}(t)>0$ for $t \geq 0$.

Now assume $u_{k} \leq r_{k}<1$. Note by definition that $u_{k}>0$. So we have

$$
\frac{u_{k}^{k+1}}{1-u_{k}}>u_{k}^{k+1} \frac{1-u_{k}^{n}}{1-u_{k}}=\sum_{i=1+k}^{n+k} u_{k}^{i} \geq 1,
$$

or equivalently $u_{k}^{k+1}+u_{k}-1>0$. But by strict upward monotonicity of $q(t)$ for $t \geq 0,0<u_{k} \leq r_{k}$ implies $u_{k}^{k+1}+u_{k}-1 \leq 0$, a contradiction. 
Plugging $u_{k}=\gamma_{k}|\theta|$ into $u_{k}>r_{k}$, we obtain an infinite family of lower bounds on zeros of $f(z)$ :

$$
|\theta|>\frac{r_{k}}{\gamma_{k}}=L_{k}, \quad k=0,1,2,3, \ldots
$$

The quantity $L_{k}$ is called the $(k+2)$-th order lower bound (and denoted by $L_{k+2}$ ) in 3 .

To obtain upper bounds on zeros of $f(z)$, we apply the above lower bounds to $g(z)=a_{0} z^{n}+a_{1} z^{n-1}+\cdots+a_{n-1} z+a_{n}$. Since the reciprocals of zeros of $g(z)$ are zeros of $f(z)$, the reciprocal of the lower bound $L_{k}$ on zeros of $g(z)$ is the upper bound $U_{k}$ on zeros of $f(z)$, which is called the $(k+2)$-th order upper bound (and denoted by $\left.U_{k+2}\right)$ in 3 .

The first bounds $L_{0}$ and $U_{0}$ of this family also appear in Henrici 1 as Theorem $6.4 \mathrm{~b}$ and Corollary $6.4 \mathrm{k}$, respectively.

Remark 1.2. A straightforward computation of the lower bound $L_{m}$ on zeros of $f(z)$ takes $O\left(m^{3} n\right)$ operations.

In Section 2, we give a simple characterization of Kalantari's bounds which leads to an efficient algorithm that computes the first $m$ bounds on zeros of $f(z)$ in $O(m n)$ operations. In Section [3, we prove that for every complex polynomial the upper and lower bounds converge to the tightest annulus containing the roots, and thus settle a problem raised in 3 .

\section{The ALGORITHM}

The key element in our algorithm is the efficient computation of $\hat{c}_{k, i}$. We start with an alternative interpretation of $\hat{c}_{k, i}$.

For each integer $k \geq 0$, define

$$
\Omega(k)=\left\{1+\sum_{i=1+k}^{n+k} \alpha_{i} z^{i}: \alpha_{i} \in \mathbb{C}\right\} .
$$

That is, $\Omega(k)$ is the set of complex polynomials of degree $n+k$ whose constant term is 1 , and the coefficients of $z^{i}, i=1, \ldots, k$ are 0 .

Then for each integer $k \geq 0$, there exists a unique polynomial $q_{k}(z)$ of degree $k$, such that

$$
f(z) q_{k}(z) \in \Omega(k) .
$$

Moreover, the coefficient of $z^{i}, i \geq 0$, in $q_{k}(z)$ is independent of $k$, so we can write

$$
q_{k}(z)=\sum_{i=0}^{k} b_{i} z^{i}
$$

The existence and uniqueness of $b_{i}, i=0,1,2, \ldots$, and hence the existence and uniqueness of $q_{k}(z), k=0,1,2, \ldots$, are implied by recurrence (2.2) shown below.

For each integer $k \geq 0$, let

$$
h_{n+k}(z) \equiv f(z) q_{k}(z)=f(z) \sum_{i=0}^{k} b_{i} z^{i}=1+\sum_{i=1+k}^{n+k} c_{k, i} z^{i} .
$$

That is, $c_{k, i}$ is defined as the coefficient of $z^{i}$ in $h_{n+k}(z)$. 
It is easy to see that $b_{i}$ and $h_{n+i}(z), i=0,1,2, \ldots$, satisfy the following recurrence:

$$
\begin{aligned}
& b_{0}=1 / a_{0}, \quad h_{n}(z)=b_{0} f(z), \\
& b_{i}=-c_{i-1, i} / a_{0}, \quad h_{n+i}(z)=h_{n+i-1}(z)+b_{i} z^{i} f(z), \quad i=1,2, \ldots
\end{aligned}
$$

Proposition 2.1. For each integer $k \geq 0$, define

$$
\hat{h}_{n+k}(z) \equiv 1+\sum_{i=1+k}^{n+k} \hat{c}_{k, i} z^{i} .
$$

Then

$$
\hat{h}_{n+k}(z) \equiv h_{n+k}(z)
$$

or equivalently $\hat{c}_{k, i}=c_{k, i}$ for $i=1+k, \ldots, n+k$.

Proof. From (1.2) the zeros of $f(z)$ are also zeros of $\hat{h}_{n+k}(z)$. Thus, if the zeros of $f(z)$ are all simple, we can factor $f(z)$ out of $\hat{h}_{n+k}(z)$. That is, $\hat{h}_{n+k}(z)=f(z) \hat{g}_{k}(z)$, where $\hat{g}_{k}(z)$ is a polynomial of degree $k$. Since $\hat{h}_{n+k}(z) \in \Omega(k)$, we have

$$
\hat{g}_{k}(z) \equiv g_{k}(z), \quad \hat{h}_{n+k}(z) \equiv h_{n+k}(z),
$$

hence the proof of the claim.

If $f(z)$ has root(s) of multiplicity greater than 1 , we can view $f(z)$ as the limit of a sequence of polynomials of degree $n$ whose roots are all simple. Since both $c_{k, i}$ and $\hat{c}_{k, i}$ are continuous functions of $a_{0}, \ldots, a_{n}$, we conclude that our claim holds in this case, too.

Now we can write $\gamma_{k}$ as a function of $c_{k, i}, i=1+k, \ldots, n+k$ :

$$
\gamma_{k}=\max \left\{\left|c_{k, i}\right|^{1 / i}: i=1+k, \ldots, n+k\right\} .
$$

Our algorithm makes use of recurrence (2.2) to compute $h_{n+k}(z)$, from which the coefficients $c_{k, i}$ are extracted to compute $\gamma_{k}$ and then the lower bounds $L_{k}, k=$ $0, \ldots, m-1$. Observe that $r_{k}$ is independent of the polynomial $f$ whose roots we want to bound, so it can be precomputed and stored in a lookup table. We shall assume $r_{k}$ is available to our algorithm.

Algorithm 1: Computing the first $m$ lower bounds on zeros of $f(z)$ in $O(m n)$ operations.

input : $f(z)=a_{n} z^{n}+a_{n-1} z^{n-1}+\cdots+a_{1} z+a_{0}, a_{n} a_{0} \neq 0$ and $m$.

output: The first $m$ lower bounds on zeros of $f(z)$.

$$
\begin{aligned}
& b_{0}=1 / a_{0} \\
& h_{n}(z)=b_{0} f(z) ; \\
& \gamma_{0}=\max \left\{\left|c_{0, i}\right|^{1 / i}: i=1, \ldots, n\right\} \\
& \text { output } L_{0}=r_{0} / \gamma_{0} ; \\
& \text { for } k \text { from } 1 \text { to } m-1 \text { do } \\
& \quad b_{k}=-c_{k-1, k} / a_{0} ; \\
& \quad h_{n+k}(z)=h_{n+k-1}(z)+b_{k} z^{k} f(z) ; \\
& \quad \gamma_{k}=\max \left\{\left|c_{k, i}\right|^{1 / i}: i=1+k, \ldots, n+k\right\} \\
& \quad \text { output } L_{k}=r_{k} / \gamma_{k} ; \\
& \text { end }
\end{aligned}
$$


Remark 2.2. We can scale $f(z)$ to make $a_{0}=1$, thus eliminating the division operation when computing $b_{i}$.

It is hard to believe that the lower bounds generated by such a simple scheme would be asymptotically sharp, not to mention that each $L_{k}$ has to be a lower bound on zeros of $q_{k}(z)=\sum_{i=0}^{k} b_{i} z^{i}$ as well. Yet extensive numerical experiments suggest that this family is indeed asymptotically sharp.

Example 2.3. Let $f(z)=3 z^{5}+4 z^{4}+4 z^{3}+2 z^{2}+2 z+1$. It has 5 distinct roots: $\theta_{1}=-0.57478, \theta_{2}=0.27086+0.69254 i, \theta_{3}=0.27086-0.69254 i, \theta_{4}=$ $-0.65014+0.79124 i, \theta_{5}=-0.65014-0.79124 i$, and the smallest and largest moduli of these roots are 0.57478 and 1.0241 , respectively.

We now use Algorithm 1 to compute the first 7 lower bounds on zeros of $f(z)$ :

\begin{tabular}{|r|r|l|l|l|l|}
\hline$k$ & $b_{k}$ & $h_{5+k}(z)$ & $\gamma_{k}$ & $r_{k}$ & $L_{k}$ \\
\hline 0 & 1 & $1+2 z+2 z^{2}+4 z^{3}+4 z^{4}+3 z^{5}$ & 2. & 0.50000 & 0.25000 \\
\hline 1 & -2 & $1-2 z^{2}-4 z^{4}-5 z^{5}-6 z^{6}$ & 1.4142 & 0.61803 & 0.43702 \\
\hline 2 & 2 & $1+4 z^{3}+3 z^{5}+2 z^{6}+6 z^{7}$ & 1.5874 & 0.68233 & 0.42984 \\
\hline 3 & -4 & $1-8 z^{4}-5 z^{5}-14 z^{6}-10 z^{7}-12 z^{8}$ & 1.6818 & 0.72449 & 0.43079 \\
\hline 4 & 8 & $1+11 z^{5}+2 z^{6}+22 z^{7}+20 z^{8}+24 z^{9}$ & 1.6154 & 0.75488 & 0.46730 \\
\hline 5 & -11 & $1-20 z^{6}-24 z^{8}-20 z^{9}-33 z^{10}$ & 1.6475 & 0.77809 & 0.47227 \\
\hline 6 & 20 & $1+40 z^{7}+16 z^{8}+60 z^{9}+47 z^{10}+60 z^{11}$ & 1.6938 & 0.79654 & 0.47027 \\
\hline
\end{tabular}

As the above table shows, although the first few bounds are not monotonically improving, the trend is already set. As we explore higher order bounds, this trend toward convergence becomes very clear:

\begin{tabular}{|r|l|l||r|l|l||r|r|l|}
\hline$k$ & $L_{k}$ & $U_{k}$ & $k$ & $L_{k}$ & $U_{k}$ & $k$ & $L_{k}$ & $U_{k}$ \\
\hline 10 & 0.49680 & 1.2140 & 150 & 0.56178 & 1.0502 & 1000 & 0.57192 & 1.0295 \\
\hline 50 & 0.54586 & 1.0838 & 200 & 0.56436 & 1.0449 & 4000 & 0.57389 & 1.0258 \\
\hline 100 & 0.55717 & 1.0598 & 250 & 0.56602 & 1.0413 & 8000 & 0.57429 & 1.0249 \\
\hline
\end{tabular}

Upper bounds for zeros of $f(z)$ given by the well-known formulas (i)-(viii) listed in Mignotte [6, Theorem 4.2, pp. 146-148] are (i) 4.3333, (ii) 2.3333, (iii, iv) 6.6667, (v) 2.6667, (vi) 1.6667 , (vii) 1.3333 , and (viii) 5.3985 . The best bound is obtained by formula (vii), which only works for polynomials with positive real coefficients. The first 3 upper bounds in Kalantari's family are $U_{0}=2.6667, U_{1}=1.6759$, and $U_{2}=1.1774$. So in this particular instance, $U_{2}$ beats all the bounds in Theorem 4.2 of [6. Moreover, Kalantari's bounds are applicable to all complex polynomials.

In general, the first members of Kalantari's family already exhibit superior performance as individual bounds. In an empirical study conducted by McNamee and Olhovsky [5], $U_{2}$ stands out as the best among a sample of upper bounds, outperforming 44 other bounds in the literature by a wide margin. However, the superiority of Kalantari's bounds are achieved at the price of high computational complexity.

A MAPLE program that implements Algorithm 1 and its upper bound counterpart is available online at http://paul.rutgers.edu/ $y_{j}$ in/MAPLE/kbounds. 


\section{Proof of Convergence}

In this section we shall prove the following result.

Theorem 3.1. Let $\theta_{1}, \ldots, \theta_{s}$ be distinct zeros of $f(z)$ with multiplicities $d_{1}, \ldots, d_{s}$; then

$$
\lim _{k \rightarrow \infty} L_{k}=\min \left\{\left|\theta_{1}\right|,\left|\theta_{2}\right|, \ldots,\left|\theta_{s}\right|\right\} .
$$

Remark 3.2. By symmetry, we also have

$$
\lim _{k \rightarrow \infty} U_{k}=\max \left\{\left|\theta_{1}\right|,\left|\theta_{2}\right|, \ldots,\left|\theta_{s}\right|\right\} .
$$

Thus, both the lower and upper bounds are asymptotically sharp.

Proof of Theorem 3.1. The key observation is that the sequence $\left\{b_{i}\right\}_{i=0}^{\infty}$ is generated by $\frac{1}{f(z)}$, i.e.,

$$
\frac{1}{f(z)}=\frac{1}{a_{n} z^{n}+a_{n-1} z^{n-1}+\cdots+a_{1} z+a_{0}}=\sum_{i=0}^{\infty} b_{i} z^{i} .
$$

To see this, note that (2.1) implies

$$
\begin{aligned}
a_{0} b_{0}=1, & \\
\sum_{j=0}^{i} a_{j} b_{i-j} & =0 \quad \text { for } 1 \leq i<n, \\
\sum_{j=0}^{n} a_{j} b_{i-j} & =0 \quad \text { for } i \geq n,
\end{aligned}
$$

so we have

$$
f(z)\left(\sum_{i=0}^{\infty} b_{i} z^{i}\right)=\left(\sum_{i=0}^{n} a_{i} z^{i}\right)\left(\sum_{i=0}^{\infty} b_{i} z^{i}\right)=1 .
$$

Remark 3.3. The above identity may be seen as the limiting case of (2.1) as $k$ approaches infinity.

It is well known in the calculus of finite differences that

$$
b_{i}=\sum_{j=1}^{s} P_{j}(i) \theta_{j}^{-i} \quad \text { for } i \geq 0
$$

where

$$
f(z)=a_{0}+a_{1} z+\cdots+a_{n-1} z^{n-1}+a_{n} z^{n}=a_{0} \prod_{j=1}^{s}\left(1-\theta_{j}^{-1} z\right)^{d_{j}}
$$

and $P_{j}(x)$ is a polynomial of degree $d_{j}-1$ (see Stanley [7, Theorem 4.1, p. 110]). This can be proved by expanding the rational function $\frac{1}{f(z)}$ into partial fractions, or by solving the linear homogeneous recurrence (3.1c).

Let $\rho_{\min }=\min \left\{\left|\theta_{1}\right|,\left|\theta_{2}\right|, \ldots,\left|\theta_{s}\right|\right\}$. First we bound $b_{i}$ :

$$
\left|b_{i}\right| \leq \sum_{j=1}^{s}\left|P_{j}(i) \theta_{j}^{-i}\right| \leq \sum_{j=1}^{s}\left|P_{j}(i)\right| \rho_{\min }^{-i} \leq Q(i) \rho_{\min }^{-i},
$$

where $Q(x)$ is a polynomial of degree $\max \left\{d_{1}, \ldots, d_{s}\right\}-1$ with positive coefficients. 
Next we bound $c_{k, i}$. We shall use the fact that $Q(x)$ is monotonically increasing when $x \geq 0$.

Let

$$
\alpha=\max \left\{\left|a_{0}\right|,\left|a_{1}\right|, \ldots,\left|a_{n}\right|\right\} \quad \text { and } \quad \beta=\max \left\{1, \rho_{\min }^{n}\right\} \sum_{j=0}^{n-1} \rho_{\min }^{j} .
$$

For $k \geq n, i=1+k, \ldots, n+k$, we have

$$
\left|c_{k, i}\right|=\left|\sum_{j=i-n}^{k} a_{i-j} b_{j}\right| \leq \alpha Q(k) \sum_{j=i-n}^{k} \rho_{\min }^{-j} \leq \alpha Q(i) \rho_{\min }^{-i+(i-k)} \sum_{j=0}^{n-1} \rho_{\min }^{j} \leq \alpha \beta Q(i) \rho_{\min }^{-i} .
$$

Thus,

$$
\begin{aligned}
\gamma_{k} & =\max \left\{\left|c_{k, i}\right|^{1 / i}: i=1+k, \ldots, n+k\right\} \\
& \leq \max \left\{[\alpha \beta Q(i)]^{1 / i}: i=1+k, \ldots, n+k\right\} \rho_{\min }^{-1}=B_{k} .
\end{aligned}
$$

On the other hand, $\gamma_{k}>r_{k} / \rho_{\min }=A_{k}$. It is easy to verify that

$$
\lim _{i \rightarrow \infty}[\alpha \beta Q(i)]^{1 / i}=1 \quad \text { and } \quad \lim _{k \rightarrow \infty} r_{k}=1 .
$$

So

$$
\lim _{k \rightarrow \infty} A_{k}=\rho_{\min }^{-1} \quad \text { and } \quad \lim _{k \rightarrow \infty} B_{k}=\rho_{\min }^{-1} .
$$

Since $A_{k}<\gamma_{k} \leq B_{k}$, we conclude that

$$
\lim _{k \rightarrow \infty} \gamma_{k}=\rho_{\min }^{-1} .
$$

Finally,

$$
\lim _{k \rightarrow \infty} L_{k}=\lim _{k \rightarrow \infty} \frac{r_{k}}{\gamma_{k}}=\rho_{\min }=\min \left\{\left|\theta_{1}\right|,\left|\theta_{2}\right|, \ldots,\left|\theta_{s}\right|\right\} .
$$

Remark 3.4. The linear recurrence (3.1c) with initial conditions (3.1a) and (3.1b) allows us to compute $b_{0}, b_{1}, \ldots, b_{k}$ directly. Together with identity (2.1), it gives rise to another efficient algorithm to compute $c_{k, i}, i=1+k, \ldots, n+k$ :

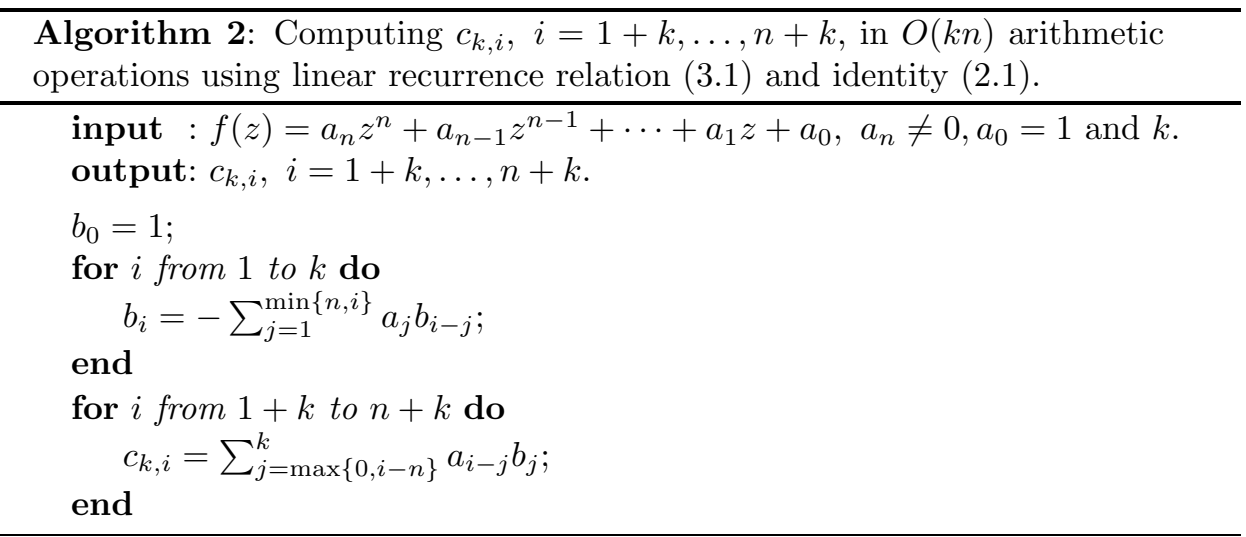


Remark 3.5. While there are plenty of individual bounds on polynomial roots in the literature, families of such bounds are rare, and Kalantari's family is the only one we know of that is asymptotically sharp. For a bibliography on bounds of polynomial roots, see McNamee [4].

\section{ACKNowledgments}

I would like to thank Professor Bahman Kalantari for supplying an early version of [3] and offering many suggestions which greatly improved the presentation of this paper.

The use of the generating function technique in our proof of convergence was inspired by a lovely short article, 2, distributed as a handout in Professor Doron Zeilberger's Experimental Mathematics class on November 15, 2004.

\section{REFERENCES}

1. P. Henrici, Applied and Computational Complex Analysis, Vol. I, Wiley, New York, 1974. MR0372162(51:8378)

2. M. D. Hirschhorn, A proof in the spirit of Zeilberger of an amazing identity of Ramanujan, Math. Mag. 69 (1996), no. 4, 267-269. MR.1424441 (97k:05019)

3. B. Kalantari, An infinite family of bounds on zeros of analytic functions and relationship to Smale's bound, Math. Comp. 74 (2005), 841-852. MR2114651(2005k:65093)

4. J. M. McNamee, A 2002 update of the supplementary bibliography on roots of polynomials, J. Comput. Appl. Math. 142 (2002), 433-434. MR1906741

5. J. M. McNamee and M. Olhovsky, A comparison of a priori bounds on (real or complex) roots of polynomials, to appear in Proceedings of 17th IMACS World Congress, Paris, France, July 2005.

6. M. Mignotte, Mathematics for Computer Algebra, translated from the French by Catherine Mignotte, Springer-Verlag, New York, 1992. MR1140923 (92i:68071)

7. R. Stanley, Generating functions, in: Studies in Combinatorics, Gian-Carlo Rota, editor, Math. Asso. Amer., 1978, pp. 100-141. MR0513004 (81i:05015)

Department of Computer Science, Rutgers University, New Brunswick, New Jersey 08901

E-mail address: yjin@paul.rutgers.edu 